\title{
BMJ Open Light cupula: the pathophysiological basis of persistent geotropic positional nystagmus
}

Takao Imai, ${ }^{1}$ Kazunori Matsuda, ${ }^{2}$ Noriaki Takeda, ${ }^{2}$ Atsuhiko Uno, ${ }^{3}$ Tadashi Kitahara, ${ }^{4}$ Arata Horii, ${ }^{5}$ Suetaka Nishiike, ${ }^{6}$ Hidenori Inohara ${ }^{1}$

To cite: Imai T, Matsuda K, Takeda N, et al. Light cupula: the pathophysiological basis of persistent geotropic positional nystagmus. BMJ Open 2014;4:e006607. doi:10.1136/bmjopen-2014006607

- Prepublication history for this paper is available online. To view these files please visit the journal online (http://dx.doi.org/10.1136/ bmjopen-2014-006607).

TI and KM contributed equally.

Received 12 September 2014 Revised 1 November 2014 Accepted 17 November 2014

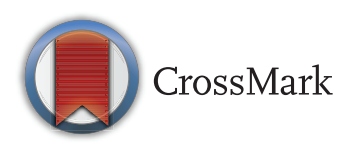

For numbered affiliations see end of article.

Correspondence to Dr Takao Imai; timai@ent. med.osaka-u.ac.jp

\section{ABSTRACT}

Objective: To clarify the pathophysiological basis of persistent geotropic positional nystagmus (PGN) in patients with the horizontal canal type of benign paroxysmal positional vertigo (H-BPPV), the time constant (TC) of nystagmus and the relationship between its slow phase eye velocity (SPV) and the angle of head rotation in supine were defined.

Methods: Geotropic or apogeotropic positional nystagmus was recorded by video-oculography and analysed three-dimensionally.

Results: Geotropic positional nystagmuses in patients with H-BPPV were classified as transient geotropic positional nystagmus with a TC of $\leq 35$ s or PGN with a TC of $>35 \mathrm{~s}$. Alternatively, the TC of persistent apogeotropic positional nystagmus (AN) in patients with H-BPPV was $>35 \mathrm{~s}$. The direction of the SPV of patients with PGN was opposite to that of patients with AN at each head position across the range of neutral head positions. The relationship between the SPV of patients with PGN and the angle of head rotation was linearly symmetrical against that of patients with AN with respect to a line drawn on the neutral head position.

Conclusions: Since its TC was $>35 \mathrm{~s}$, it is suggested that PGN is induced by cupula deviation in response to gravity at each head position. It is also suggested that the direction of cupula deviation in patients with PGN is opposite to that of patients with AN across the neutral head positional range with no nystagmus where the long axis of cupula is in alignment with the axis of gravity. Since the pathophysiological basis of AN is considered a heavy cupula, it is suggested that PGN is conversely induced by a light cupula.

\section{INTRODUCTION}

Benign paroxysmal positional vertigo (BPPV) is the most common peripheral vestibular disease, and it is usually caused by involvement of the posterior and/or horizontal semicircular canal (HSCG). ${ }^{1}$ In particular, horizontal positional nystagmus in the supine position of patients with the HSCC type of BPPV (H-BPPV) consists of the geotropic type (fast phase towards the ground) as well as the apogeotropic type (fast phase

\section{Strengths and limitations of this study}

- In this study, the benign paroxysmal positional nystagmus was precisely analysed in three dimensions.

- This is the first study to clarify the relationship between slow phase eye velocity of persistent geotropic positional nystagmus and the angle of head rotation.

- The light cupula theory in this study was discussed under the assumption that apogeotropic positional nystagmus is caused by a heavy cupula.

away from the ground). ${ }^{2}$ Geotropic positional nystagmus is induced by canalolithiasis-freefloating debris-in HSCG patients when the head is rotated to either side in a supine position. $^{3}$ Thus, transient geotropic positional nystagmus (TGN) disappears rapidly when the head position is maintained. However, in HSCC patients, apogeotropic positional nystagmus is induced by a deviation of the cupula and attached debris, known as cupulolithiasis, in response to the head position. ${ }^{4}$ Thus, persistent apogeotropic positional nystagmus (AN) lasts longer than TGN even when the head position is maintained. ${ }^{5}$

Recently, another type of geotropic nystagmus-persistent geotropic positional nystagmus (PGN) - has been reported in patients with H-BPPV. ${ }^{6}$ In the present study, an attempt was made to clarify the pathophysiology of PGN in patients with H-BPPV. We examine the time constant (TC) of this type of nystagmus and the relationship between its slow phase eye velocity (SPV) and the angle of head rotation in a supine position.

\section{METHODS \\ Patients}

This study includes a total of 107 patients with H-BPPV who visited the Department of Otorhinolaryngology-Head and Neck 
Surgery of the Osaka University Hospital between 15 February 2011 and 31 May 2013, reporting positional vertigo, showing geotropic or apogeotropic positional nystagmus and having no central lesion identified by MRI. Written informed consent was obtained from all patients before the study, which was performed in line with the Helsinki II Declaration. The study is also reported in accordance with the guidelines of standards for the reporting of diagnostic accuracy studies (STARD). The patients who could not consent to this study, those who had additional positional nystagmus of the posterior canal type of BPPV (P-BPPV) and those who showed transition from apogeotropic nystagmus to geotropic nystagmus were excluded (figure 1).

The patients lay in the supine position and their heads were rotated quickly or stepwise. Their positional nystagmus was then recorded by video-oculography $(720 \times 480 \mathrm{dot}, \quad 30 \mathrm{~Hz})$ using RealEyes (Micromedical Technologies). The angle of the head in the supine position and the rotational velocity around threedimensional axes of WAA-006 sensors (ATR-Promotions) were also recorded with a sensor of linear acceleration in three dimensions.

\section{Analysis of head position}

To record the angle of head rotation, WAA-006 sensors were attached to RealEyes goggles and the sensors' $\mathrm{X}, \mathrm{Y}$ and $\mathrm{Z}$ axes were aligned with the patient's nasooccipital, interaural and dorsoventral axes, respectively. The head position was calculated by applying the atan2 function in Excel software (Microsoft) to the WAA-006 data (atan2(z, y)). WAA-006 sensors can record the information of the computer timer. Using this information, the data of WAA-006 sensors were synchronised to the video of eye movement.

\section{Analysis of positional nystagmus and SPV of positional nystagmus}

Positional nystagmus was recorded on a Windows computer with the goggles. The digital movie of positional nystagmus was converted to $720 \times 480 \mathrm{dot}$ Jpeg images and analysed using an algorithm. ${ }^{7}$ In this study, eye

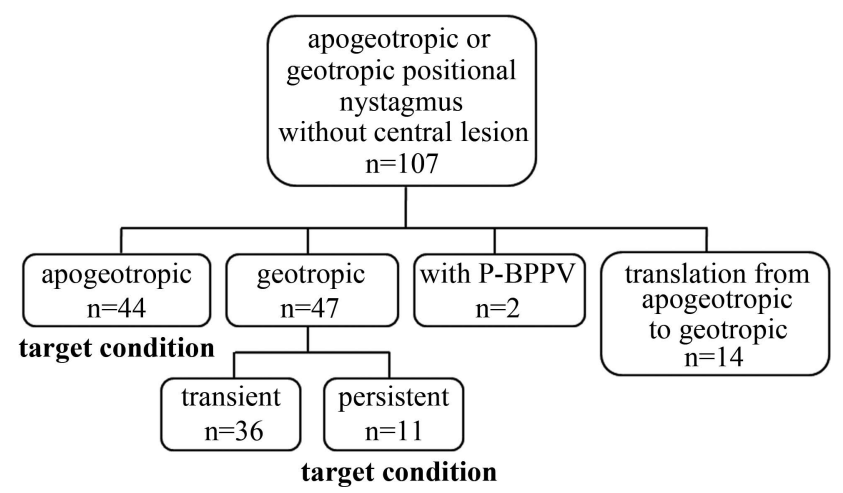

Figure 1 Classification of patients (P-BPPV, posterior canal type of benign paroxysmal positional vertigo). movements were three-dimensionally described by rotation vectors, which characterise the eye positions around a single axis. An eye position can be reached by rotating the eye from the reference position around a single axis. The eye position is represented by a vector around the axis, the length of which is proportional to the angle of rotation. The reference position was defined as the eye position when the patient was looking straight ahead with the head in an upright position. Straight ahead was defined as looking at a target located horizontally in front of the eye. ${ }^{8}$ The method of analysing the eye rotation vector and its accuracy have already been described elsewhere. ${ }^{79}$ The spatial coordinates of the pupil centre and an iris freckle were reconstructed in three dimensions and were defined as follows: the $\mathrm{X}$ axis, parallel to the nasooccipital axis (positive forward); the Y axis, parallel to the interaural axis (positive left) and the $\mathrm{Z}$ axis, normal to the X-Y plane (positive upwards). The X, Y and $\mathrm{Z}$ components mainly reflect the roll, pitch and yaw components, respectively. The direction of rotation was described from the patient's point of view. Regarding the $\mathrm{X}$ component, 'right torsional' indicates that the superior pole of the eyeball rotates towards the right ear and "left torsional" indicates rotation towards the left ear. The rotation vector $\mathbf{r}$, describing a rotation of $\theta$ around the axis $\mathbf{n}$, was given by the formula $\mathbf{r}=\mathbf{n} \tan (\theta / 2)$, with $\mathbf{n}$ being the unit vector, whose direction represents its axis. We used the Euler angle parameter that was given as $2 \times \tan ^{-1}$ (magnitude of rotation vector) to represent the eye position as an axis angle. ${ }^{10}{ }^{11}$ Using the following formula: $\boldsymbol{\omega}=2 \times(\mathrm{d} \mathbf{r} / \mathrm{dt}+\mathbf{r} \times \mathrm{d} \mathbf{r} / \mathrm{dt}) /\left(1+\mathbf{r}^{2}\right)$, we calculated the eye velocity $\omega$ around the $\mathrm{X}, \mathrm{Y}$ and $\mathrm{Z}$ axes. ${ }^{8}$ We then extracted the SPV of the nystagmus by a fuzzy set-based approach. ${ }^{1213}$

\section{Calculating TC}

All patients in this study showed rightward and leftward horizontal nystagmus because the nystagmus was either geotropic or apogeotropic. When calculating TC, we used the rightward positional nystagmus when the SPV of rightward nystagmus was larger than that of leftward nystagmus, and vice versa. We based our calculations on a $30 \mathrm{~s}$ period from the time that the SPV reached its maximum value. When the direction of nystagmus along the $\mathrm{Z}$ component was reversed within the $30 \mathrm{~s}$ period, we used the data from the time that the SPV reached the maximum value to the time just before the sign of the $\mathrm{Z}$ component of the SPV changed. Using the least squares method, the SPV against time in three components was approximated exponentially. Finally, TC was calculated as the reciprocal of the coefficient of time. ${ }^{14}$

\section{Approximating a formula describing the relationship}

\section{between SPV and head rotation angle in supine subjects}

We excluded the data reflecting the vestibulo-ocular reflex during head movement from the data of apogeotropic or geotropic nystagmus with a long TC and used the remaining data to plot the $\mathrm{Z}$ component of the SPV on the $\mathrm{Y}$ axis 
and the head position on the $\mathrm{X}$ axis. Using the least squares method, the plotted data were approximated by the following formula: $\alpha \sin (x-\theta) \quad(x \geq \theta), \beta \sin (x-\theta) \quad(x<\theta)$. The data of this function between $-90^{\circ}$ and $90^{\circ}$ were approximated by quartic function, because this function is non-differentiable at point $\mathrm{x}=\theta$ but is continuous.

\section{RESULTS}

A total of 107 eligible patients with H-BPPV (22 men and 85 women, median age 68 years, age range $36-88$ years) who reported positional vertigo and showed geotropic or apogeotropic positional nystagmus were included in this study (figure 1). We excluded 2 patients who had an additional positional nystagmus of type P-BPPV, and 14 patients who showed a transition from apogeotropic nystagmus to geotropic nystagmus. As shown in figure 1, of the 107 patients, apogeotropic positional nystagmus was seen in 44 patients with H-BPPV (10 men and 34 women, median age 70.5 years, age range $36-88$ years) and geotropic positional nystagmus in 47 patients with H-BPPV (10 men and 37 women, median age 67 years, age range 39-82 years). The three-dimensional eye position and SPV of PGN in patient A (a 44-year-old woman) is shown in figures 2A and 3A. A left-torsional and leftward horizontal nystagmus was induced by head rotation to the left and a right-torsional and rightward horizontal nystagmus was induced by head rotation to the right (figure 2A). The left-torsional and leftward horizontal nystagmus at the left-ear-down head position declined very gradually and was calculated by the following formulas:

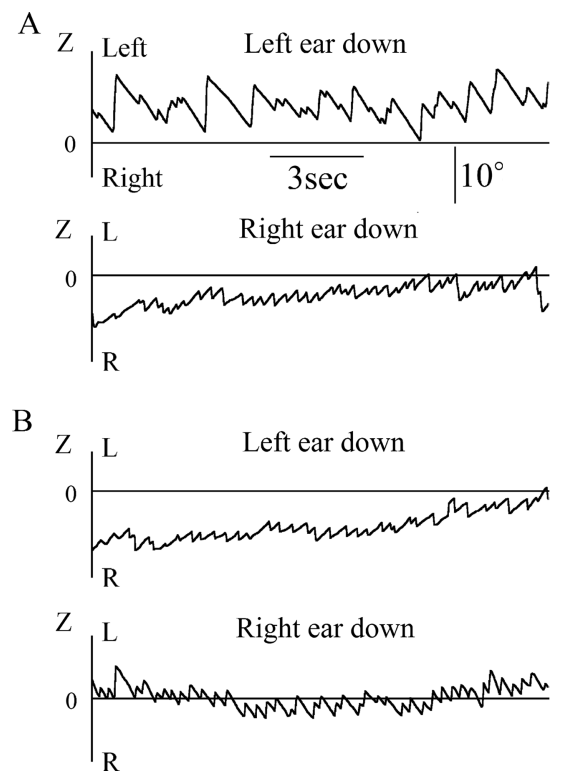

Figure 2 Eye position of positional nystagmus in the right-ear-down and left-ear-down head positions in patients with horizontal canal type of benign paroxysmal positional vertigo. (A) Persistent geotropic positional nystagmus in patient $A$ and $(B)$ persistent apogeotropic positional nystagmus in patient $B$.
$3.9 \exp \left(\left(\mathrm{t}_{1}-\mathrm{t}\right) / 121\right)$ in the $\mathrm{X}$ component, $1.9 \exp \left(\left(\mathrm{t}_{1}-\mathrm{t}\right) / 121\right)$ in the $\mathrm{Y}$ component and $-9.0 \exp \left(\left(\mathrm{t}_{1}-\mathrm{t}\right) / 121\right)$ in the $\mathrm{Z}$ component $\left(\mathrm{t}_{1}\right.$ represents the time when SPV was at a maximum), with a TC of $121 \mathrm{~s}$. The maximum SPV of lefttorsional and leftward horizontal nystagmus at the left-ear-down head position was higher than that of the right-torsional and rightward horizontal nystagmus induced in the right-ear-down head position (figure 3A). The three-dimensional eye position and SPV of AN in patient B (a 74-year-old woman) are shown in figures 2B and 3B. A left-torsional and leftward horizontal nystagmus was induced by head rotation to the right while a right-torsional and rightward horizontal nystagmus was induced by head rotation to the left (figure 2B). The left-torsional and leftward horizontal nystagmus at the right-ear-down head position declined very gradually and was calculated by the following formulas: $1.8 \exp \left(\left(\mathrm{t}_{4}-\mathrm{t}\right) / 2897\right)$ in the $\mathrm{X}$ component, $-0.3 \exp$ $\left(\left(t_{4}-t\right) / 2897\right)$ in the $Y$ component and $-5.3 \exp \left(\left(t_{4}-t\right) / 2897\right)$ in the $\mathrm{Z}$ component $\left(\mathrm{t}_{4}\right.$ represents the time when SPV was maximum), with a TC of $2897 \mathrm{~s}$. The maximum SPV of the left-torsional and leftward horizontal nystagmus in the right-ear-down head position was higher than that of the right-torsional and rightward horizontal nystagmus in the left-ear-down head position (figure 3B).

The TC of patients with TGN ranged from 4.9 to $32.2 \mathrm{~s}$ (median: $11.7 \mathrm{~s}$ ). The distribution of TCs of 11 patients with PGN and of 44 patients with AN is shown in figure 4 . The TCs of patients with PGN ranged from 66 to $3600 \mathrm{~s}$ (median: $168 \mathrm{~s}$ ). The TCs of patients with AN ranged from 35.3 to $3600 \mathrm{~s}$ (median: $127 \mathrm{~s}$ ). Changes in the SPV induced by stepwise rotation in patient A with PGN are shown in figure 5A. Rightward nystagmus was induced when the head was rotated $61^{\circ}$ to the right and gradually disappeared with the stepwise rotation of the head to the left. Leftward nystagmus was then induced by further stepwise rotation of the head to the left. The SPV of rightward and leftward nystagmus changed stepwise in accordance with the stepwise rotation of the head. However, no nystamus was induced when the head was rotated $24^{\circ}$ to the left to a neutral head position. Changes in the SPV induced by stepwise rotation in patient $\mathrm{B}$ with $\mathrm{AN}$ are shown in figure 5B. Rightward nystagmus was induced when the head was rotated $57^{\circ}$ to the left and gradually disappeared with stepwise rotation of the head to the right. Leftward nystagmus was then induced by further stepwise rotation to the right. The SPV of rightward and leftward nystagmus changed stepwise in accordance with the stepwise rotation of the head. However, no nystagmus was induced when the head was rotated $27^{\circ}$ to the left to a neutral head position. The relationship between the angle of head rotation and the SPV induced by stepwise head rotation in patient $\mathrm{A}$ with PGN (shown in figure 5A) was plotted in XY coordinates, with the SPV plotted on the $\mathrm{Y}$ axis against the angle of head rotation on the $\mathrm{X}$ axis (figure 6A). The relationship was approximated by the following formula: 
Figure 3 Three-dimensional $X$,

$Y$ and $Z$ components of eye velocity of positional nystagmus in right-ear-down and left-ear-down head positions in patients with horizontal semicircular canal type of benign paroxysmal positional vertigo. (A) Persistent geotropic positional nystagmus lasted with long TCs (time constant) of $121 \mathrm{~s}$ and $>1 \mathrm{~h}$ in patient $A$ and (B) $A N$ lasted with long TCs of 110 and $2897 \mathrm{~s}$ in patient $B$.
A
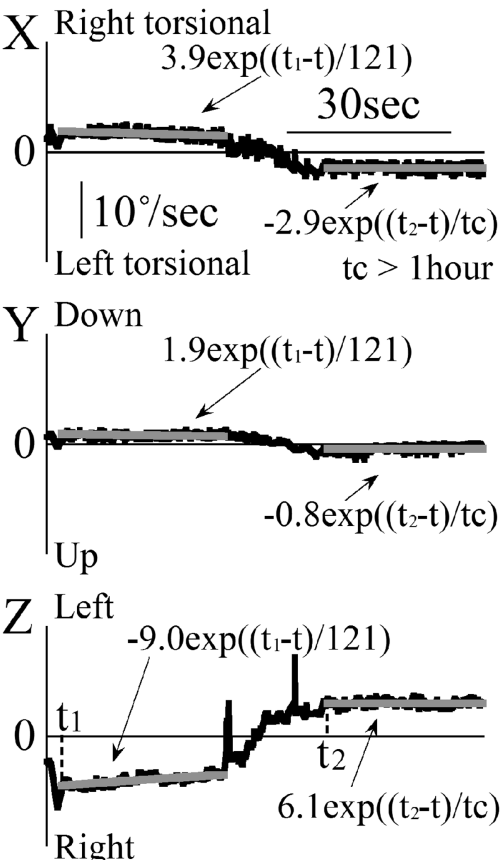

left ear down

\section{right ear}

B

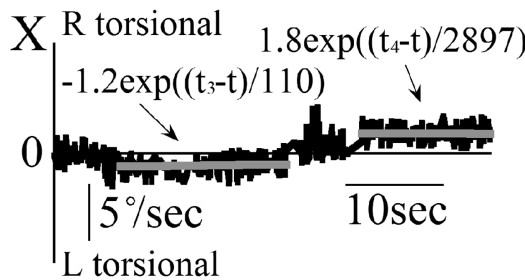

Y D
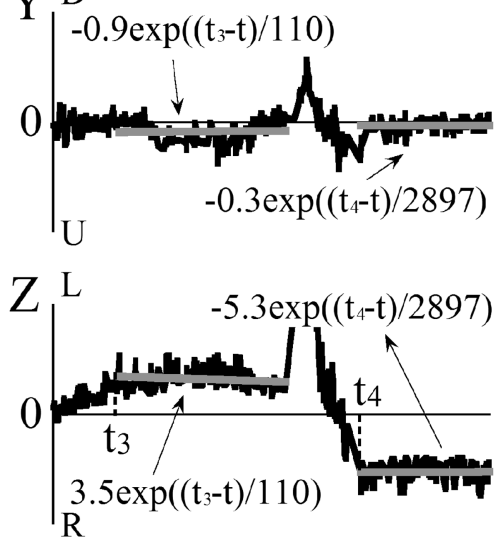

left ear right ear down $\mathrm{y}=8.9 \sin (\mathrm{x}+149.0) \quad\left(\mathrm{x} \geq 31.0^{\circ}\right), \quad 4.1 \sin (\mathrm{x}+149.0) \quad\left(\mathrm{x}<31.0^{\circ}\right)$, where $\mathrm{x}$ is the Euler angle. Values of $\mathrm{x}$ between $-90^{\circ}$ and $90^{\circ}$ were approximated by the quartic function

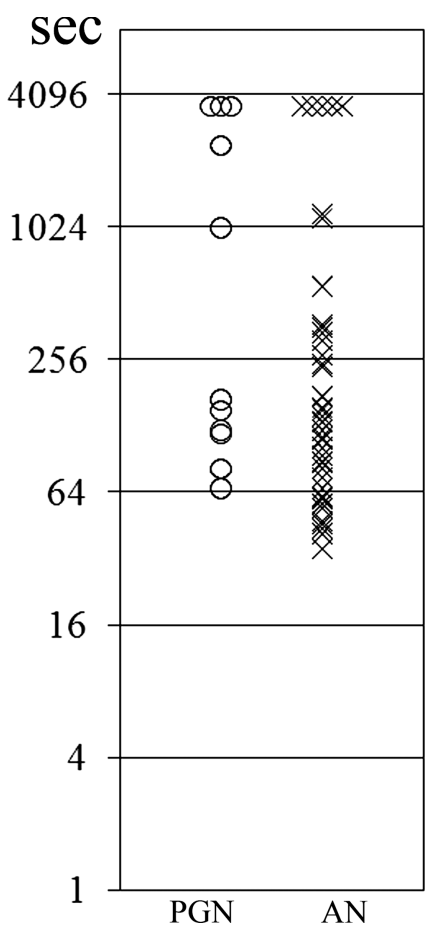

Figure 4 Distribution of time constants (TCs) of positional nystagmus in 11 patients with persistent geotropic positional nystagmus (PGN) and 44 patients with AN. TCs of all 44 patients with AN was more than $35 \mathrm{~s}$. TCs of 11 patients with PGN was more than $35 \mathrm{~s}$. When TC was more than 1 hour, we set the TC value at 3600 s.O: TC of PGN, x: TC of AN. $y=2 \times 10^{-8} x^{4}+4 \times 10^{-7} x^{3}-0.000 x^{2}-0.068 x+2.257$, where $x$ is the Euler angle, or $y=0.236 \mathrm{x}^{4}+0.073 \mathrm{x}^{3}-2.357 \mathrm{x}^{2}-3.918 \mathrm{x}$ +2.257 , where $x$ is the angle in radians.
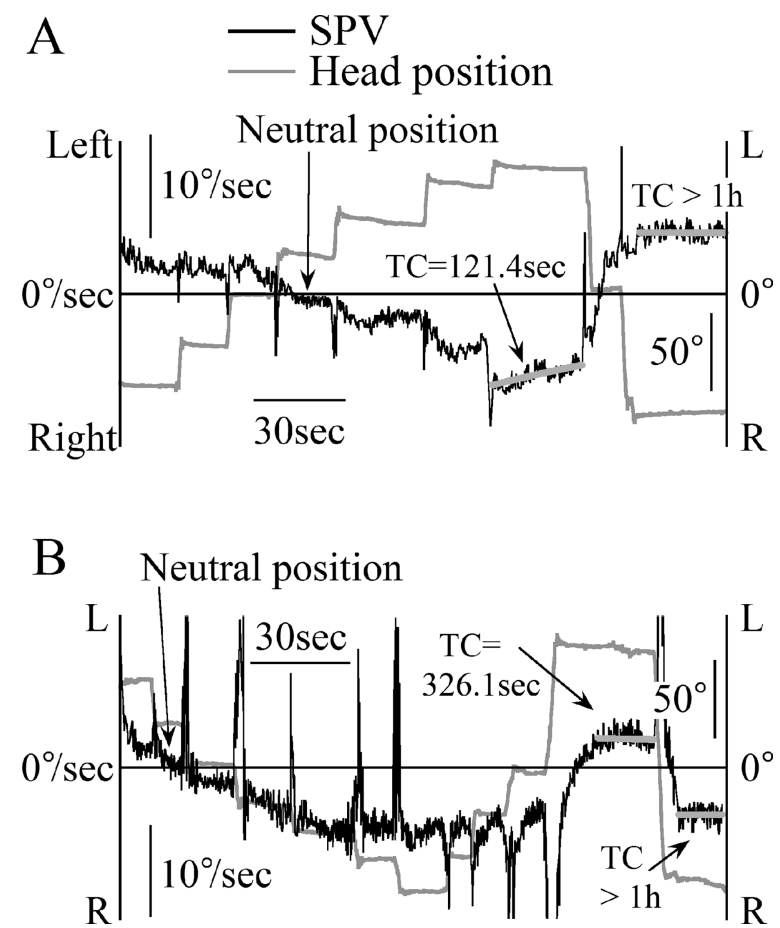

Figure 5 Changes in the slow phase eye velocity (SPV) of positional nystagmus induced by stepwise rotation of the head in patients with horizontal canal type of benign paroxysmal positional vertigo. (A) Persistent geotropic positional nystagmus in patient $A$ and $(B) A N$ in patient $B$ (TCs, time constant). 
The relationship between the angle of head rotation and the SPV induced by stepwise head rotation in patient $\mathrm{B}$ with AN (shown in figure $5 \mathrm{~B}$ ) was plotted in $\mathrm{XY}$ coordinates, with the SPV plotted on the Y axis against the angle of head rotation on the $\mathrm{X}$ axis (figure $6 \mathrm{~B}$ ). The relationship was approximated by the following formula:
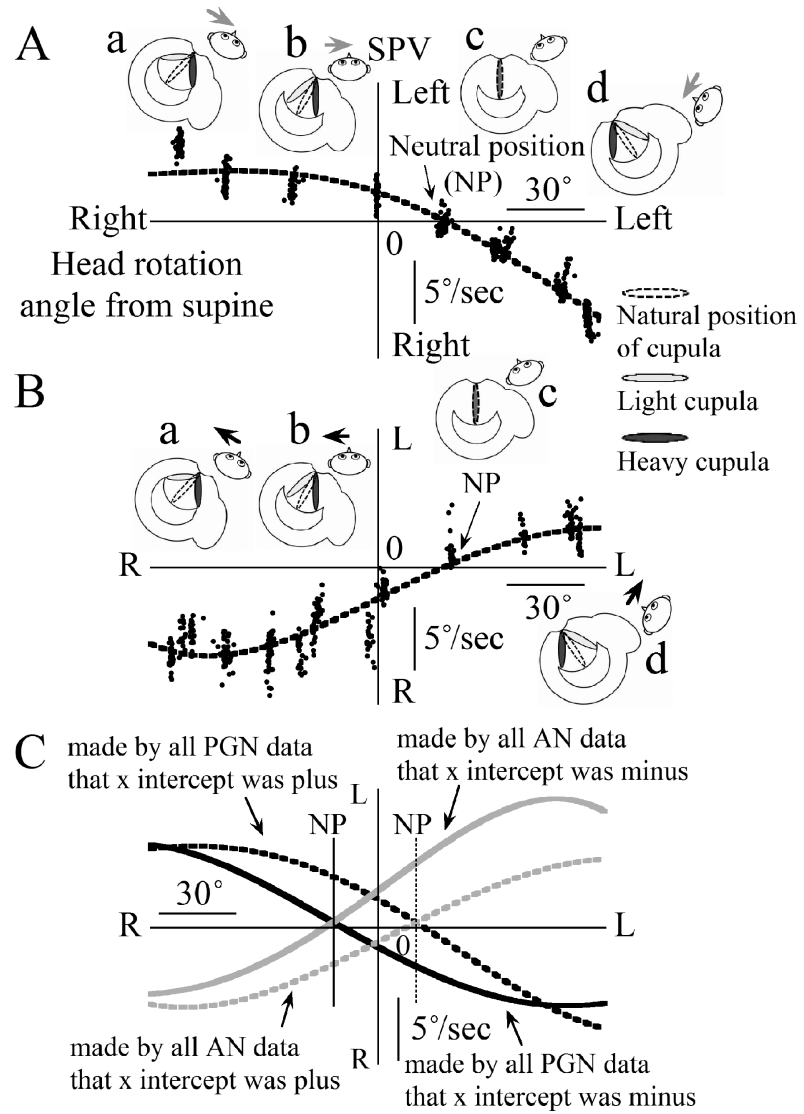

Figure 6 The relationship between the angle of head rotation and the slow phase eye velocity (SPV) of positional nystagmus in patients with horizontal canal type of benign paroxysmal positional vertigo. (A) The relationship between the angle of head rotation and the SPV of persistent geotropic positional nystagmus (PGN) induced by stepwise rotation of the head in patient $A$ as shown in figure $5 \mathrm{~A}$ was plotted in $\mathrm{XY}$ coordinates on the $Y$ axis against the angle of head rotation on the $\mathrm{X}$ axis, (B) The relationship between the angle of head rotation and the SPV of AN induced by stepwise rotation of the head in patient $B$ as shown in figure 5B was plotted in $X Y$ coordinates on the $Y$ axis against the angle of head rotation on the $X$ axis. Inserted figures: light cupula and heavy cupula in the left horizontal semicircular canal. a: Head turning to the right, b: in the supine position, $\mathrm{c}$ : in the neutral head position and $d$ : head turning to the left. Arrows near face: the direction of positional nystagmus. (C) The approximated relationship between the angle of head rotation and the SPV of PGN at the leftward neutral head position (black dotted line) and at the rightward neutral head position (black solid line). The approximated relationship between the angle of head rotation and the SPV of AN in the leftward neutral head position (grey dotted line) and in the rightward neutral head position (grey solid line). $y=3.6 \sin (x-22.0) \quad\left(x \geq 22.0^{\circ}\right), 7.0 \sin (x-22.0) \quad\left(x<22.0^{\circ}\right)$ where $\mathrm{x}$ is the Euler angle. Values of $\mathrm{x}$ between $-90^{\circ}$ and $90^{\circ}$ were approximated by quartic function $y=2 \times 10^{-8} x^{4}-6 \times 10^{-6} x^{3}+2 \times 10^{-8} x^{2}+0.100 x-2.545$, where $x$ is the Euler angle, or $y=0.165 x^{4}-1.128 x^{3}+7 \times 10^{-5} x^{2}+5.747 x-2.545$, where $\mathrm{x}$ represents the angle in radians.

In 7 of the 11 patients with PGN, the relationship had positive x-intercepts, which indicates the absence of nystagmus at the neutral head position when the head had been rotated $15-20^{\circ}$ to the left. The relationship was approximated by the formulas $y=4 \times 10^{-8} x^{4}-8 \times 10^{-6} x^{3}-0.001 x^{2}-0.208 x+4.040$, where $x$ is the Euler angle, and $\mathrm{y}=0.481 \mathrm{x}^{4}+1.457 \mathrm{x}^{3}-3.519 \mathrm{x}^{2}-11.95 \mathrm{x}+4.040$, where $\mathrm{x}$ represents the angle in radians. The approximated relationship between the angle of head rotation and the SPV of PGN in these seven patients is shown by a black dotted line in figure 6C. However, in the other four patients, the relationship had negative x-intercepts, indicating the absence of nystagmus at the neutral head position when the head had been rotated $15-20^{\circ}$ to the right. The relationship was approximated by the formulas $y=-2 \times 10^{-8} x^{4}-8 \times 10^{-6} x^{3}-0.000 x^{2}-0.196 x-2.936$, where $x$ is the Euler angle, and $\mathrm{y}=-0.214 \mathrm{x}^{4}+1.592 \mathrm{x}^{3}+1.901 \mathrm{x}^{2}-11.28 \mathrm{x}-2.936$, where $\mathrm{x}$ represents the angle in radians. The approximated relationship between the angle of head rotation and the SPV of PGN in these four patients is shown by a black solid line in figure $6 \mathrm{C}$.

The relationship between the angle of head rotation and the SPV of 44 patients with AN with a TC of $>35 \mathrm{~s}$ was approximated by quartic function. In 23 of the 44 patients, the relationship had positive $\mathrm{x}$-intercepts, which indicates the absence of nystagmus at the neural head position when the head had been rotated $15-20^{\circ}$ to the left. The relationship was approximated by the formulas $\mathrm{y}=9 \times 10^{-9} \mathrm{x}^{4}-9 \times 10^{-6} \mathrm{x}^{3}+0.000 \mathrm{x}^{2}+0.189 \mathrm{x}-2.163$, where $x$ represents the Euler angle, and $\mathrm{y}=0.097 \mathrm{x}^{4}-1.655 \mathrm{x}^{3}+0.360 \mathrm{x}^{2}+10.84 \mathrm{x}-2.163$, where $\mathrm{x}$ represents the angle in radians. The approximated relationship between the angle of head rotation and the SPV of $\mathrm{AN}$ in these 23 patients is shown by a grey dotted line in figure $6 \mathrm{C}$. In the other 21 patients, the relationship had negative x-intercepts, indicating an absence of nystagmus at the neutral head position when the head had been rotated $15-20^{\circ}$ to the right. The relationship was approximated by the formulas $\mathrm{y}=-5 \times 10^{-8} \mathrm{x}^{4}-1 \times 10^{-5} \mathrm{x}^{3}+0.000 \mathrm{x}^{2}+0.268 \mathrm{x}+5.376$, where $\mathrm{x}$ is the Euler angle, and $\mathrm{y}=-0.556 \mathrm{x}^{4}-2.790 \mathrm{x}^{3}$ $+0.622 x^{2}+15.35 x+5.376$, where $x$ represents the angle in radians. The approximated relationship between the angle of head rotation and the SPV of these 21 patients with $\mathrm{AN}$ is shown by a grey solid line in figure 6C. The dashed black lines show the approximated relationship between the angle of head rotation and the SPV of patients with PGN with a TC of $>35 \mathrm{~s}$, which was linearly symmetrical against the dashed grey line showing the approximated relationship between the 
angle of head rotation and the SPV of patients with AN with respect to a line on positive $\mathrm{x}$-intercepts of the neutral head position (figure 6C). The solid black line shows the approximated relationship between the angle of head rotation and the SPV of patients with PGN with a TC of $>35 \mathrm{~s}$, which was linearly symmetrical against the solid grey line showing the approximated relationship between the angle of head rotation and the SPV of patients with AN with respect to a line on negative $\mathrm{x}$-intercepts of the neutral head position (figure 6C).

\section{DISCUSSION}

In this study, three-dimensional analysis of positional nystagmus showed that the TCs of PGN and AN were longer than those of TGN in patients with H-BPPV. The distributions of TCs of geotropic positional nystagmus in patients with H-BPPV were segregated into two groups: patients with PGN with TCs of $>35 \mathrm{~s}$ and patients with TGN with TCs of $\leq 35 \mathrm{~s}$. In contrast, the TCs of patients with AN were distributed over $35 \mathrm{~s}$ (figure 4). These findings suggest that PGN and AN have a common pathophysiology underlying positional nystagmus which is different from that of TGN. Since TGN is induced by canalolithiasis in HSCC and AN is induced by cupulolithiasis in HSCC, it is also suggested that a lesioned HSCC cupula induces PGN in patients with H-BPPV.

In this study, the relationship between the angle of head rotation and the SPV of positional nystagmus was quantitatively examined in patients with H-BPPV. PGN and $\mathrm{AN}$ were induced by stepwise rotation of the head (figure 5). Moreover, in PGN and AN, no nystagmus was induced in the neutral head position (figures 5 and $6 \mathrm{~A}$, B). These findings support the hypothesis that PGN is induced by a lesioned cupula in HSCC, as with the cupulolithiasis of HSCC in patients with AN. However, the direction of PGN was opposite to that of AN. Indeed, at the right-ear-down head position, right-beating nystagmus was induced in patients with PGN, while left-beating nystagmus was induced in patients with AN. In the left-ear-down head position, left-beating nystagmus was induced in patients with PGN, while right-beating nystagmus was induced in patients with AN (figures 2 and 3). Therefore, it is suggested that the response of the lesioned HSCC cupula to the head position in patients with PGN was opposite to that in those with AN.

The approximated relationship between the angle of head rotation and the SPV of PGN induced by a stepwise rotation of the head was linearly symmetrical with that of AN with respect to a line plotting the neutral head position in patients with H-BPPV (figure 6). Moreover, the approximated relationship between the angle of head rotation and the SPV of 4 patients with PGN was linearly symmetrical with the SPV of 21 patients with AN with respect to a line plotting the neutral head position where the head was rotated approximately $18^{\circ}$ to the right (figure 6C). The approximated relationship between the angle of head rotation and the SPV of 7 patients with PGN was linearly symmetrical with that of 23 patients with AN with respect to a line plotting the neutral head position where the head was rotated approximately $18^{\circ}$ to the left (figure 6C). Since the abnormally heavy cupulolithiasis deviates in response to gravity in patients with $\mathrm{AN}$, these findings suggest that the lesioned cupula deviates in an opposite direction in response to gravity in patients with PGN (inserted figures in figure $6 \mathrm{~A}, \mathrm{~B}$ ). Moreover, in the neutral head position where the head was rotated approximately $18^{\circ}$ to either the right or the left, the long axis of the HSCC cupula was in alignment with the gravitational axis, and no deviation of either the heavy or the lesioned cupula was induced (inserted ' $c$ ' in figure 6A, B). Altogether, these findings suggest that a lesioned HSCC cupula acts as a light cupula that deviates to the opposite direction of a heavy cupula in response to gravity and induces PGN in a neutral head position in patients with H-BPPV. Previous studies hypothesised that the characteristics of PGN are due to a light cupula. ${ }^{6}{ }^{15}{ }^{16}$ In this study, however, quantitative three-dimensional analysis of the response of patients with PGN to changes in head position demonstrated that lesioned HSCC cupulae deviate similarly to light cupulae in response to gravity and induced PGN in patients with H-BPPV. Bergenius and Ichijo have also speculated that the attachment of debris of lower density makes a cupula in HSCC lighter than the endolymph. ${ }^{6}{ }^{15} 16$ Alternatively, a substance in the blood, such as alcohol, which has lower density than the endolymph, could diffuse into the cupula earlier than the endolymph by way of its proximity to capillaries, rendering the cupula lighter than the endolymph. ${ }^{16}$ The attachment of debris of lower density is a feasible explanation because usually only one side is affected.

Since the cupula is laterally tilted in the supine position (inserted ' $b$ ' in figure 6A, B), a slight head rotation to the affected side while in this position can align the axis of light and heavy cupulae (inserted ' $\mathrm{c}$ ' in figure $6 \mathrm{~A}, \mathrm{~B}$ ). Four patients with PGN and 21 patients with AN reported a neutral head position when the head was rotated to the right. The right side in these patients was affected. However, the left side was affected in 7 patients with PGN (including patient A) and 23 patients with AN (including patient B) who reported a neutral head position when the head was rotated to the left. Based on Ewald's second Law, ${ }^{15}$ the flow of endolymph towards the ampulla of HSCC results in greater stimulation than flow away from the ampulla. When the left side is affected, a head rotation to the left away from the perceived neutral head position (inserted ' $c$ ' in figure 6A, B) results in a deviation of light cupulae in the ampullopetal direction (inserted ' $d$ ' in figure $6 \mathrm{~A}$ ) and heavy cupulae in the ampullofugal direction (inserted ' $d$ ' in figure 6B). As shown in figure 6A, B, in patient $\mathrm{A}$, who presents with a light left cupula, head rotation to the left (affected side) from the neutral head position caused a greater SPV than head rotation to the right (healthy side) $(8.9 \sin (\mathrm{x}+149)$ vs $4.1 \sin (\mathrm{x}+149))$. In 
patient B, who presented with a heavy left cupula, head rotation to the left (affected side) from the neutral head position caused a lesser SPV than head rotation to the right (healthy side) $(3.6 \sin (x-22.0)$ vs $7.0 \sin (x-22.0))$.

Geotropic positional nystagmus was subjectively subdivided into two groups-TGN and PGN-based on the length of the sustained period of positional nystagmus. In this study, we showed that the TC of the SPV of positional nystagmus is an objective index of transient or persistent positional nystagmus and proposed that a TC of $<35 \mathrm{~s}$ indicates a transient type of PGN while a TC of $>35 \mathrm{~s}$ indicates the persistent type.

In conclusion, in this study, we showed that the direction of the SPV of PGN with a TC of $>35 \mathrm{~s}$ was opposite to that of $\mathrm{AN}$ with a TC of $>35 \mathrm{~s}$ in response to the same neutral head position where no nystagmus was induced, and that the relationship between the SPV of PGN and the angle of head rotation was linearly symmetrical to that of AN with respect to a line plotting the neutral head position. These findings suggest that the direction of cupula deviation in PGN is opposite to that of AN across the neutral head position, where the long axis of the cupula is in alignment with the gravitational axis. Since AN was induced by a heavy cupula, we concluded that the basic pathophysiological mechanism of PGN is a light cupula in patients with H-BPPV. This light cupula theory is important for the explanation of morbidity associated with H-BPPV.

\section{Author affiliations}

${ }^{1}$ Department of Otorhinolaryngology—Head and Neck Surgery, Osaka University Graduate School of Medicine, Osaka, Japan

${ }^{2}$ Department of Otolaryngology, University of Tokushima School of Medicine, Tokushima, Japan

${ }^{3}$ Department of Otolaryngology, Osaka General Medical Center, Osaka, Japan

${ }^{4}$ Department of Otolaryngology, Nara Medical University, Nara, Japan

${ }^{5}$ Department of Otolaryngology, Osaka National Hospital, Osaka, Japan

${ }^{6}$ Department of Otolaryngology, Osaka Rosai Hospital, Osaka, Japan

Acknowledgements The professional editing service, 'American Journal Experts', helped the authors to revise their manuscript.

Contributors TI contributed to conception, acquisition of data, analysis and interpretation of data, and drafting of the article. KM contributed to analysis and interpretation of data and drafting of the article. NT contributed to design and interpretation of data, study supervision and drafting of the article. AU and TK contributed to the acquisition and interpretation of data. AH contributed to the interpretation of data. SN contributed to the interpretation of data. HI contributed to study supervision.

Funding This study was supported by JSPS KAKENHI Grant Number 24592546.
Competing interests None.

Patient consent Obtained.

Ethics approval This study was approved by the Ethics Committee of Osaka University Hospital, Osaka, Japan (No. 10091).

Provenance and peer review Not commissioned; externally peer reviewed.

Data sharing statement No additional data are available.

Open Access This is an Open Access article distributed in accordance with the Creative Commons Attribution Non Commercial (CC BY-NC 4.0) license, which permits others to distribute, remix, adapt, build upon this work noncommercially, and license their derivative works on different terms, provided the original work is properly cited and the use is non-commercial. See: http:// creativecommons.org/licenses/by-nc/4.0/

\section{REFERENCES}

1. Brandt $\mathrm{T}$, ed. Benign paroxysmal positioning vertigo. In: Vertigo: its multisensory syndromes. 2nd edn. London: Springer-Verlag, 1999:251-83.

2. Parnes LS, Agrawal SK, Atlas J. Diagnosis and management of benign paroxysmal positional vertigo (BPPV). CMAJ 2003;169:681-93.

3. Nuti D, Vannucchi P, Pagnini P. Benign paroxysmal positional vertigo of the horizontal canal: a form of canalolithiasis with variable clinical features. J Vestib Res 1996;6:173-84.

4. Bisdorff AR, Debatisse D. Localizing signs in positional vertigo due to lateral canal cupulolithiasis. Neurology 2001;57:1085-8.

5. Imai T, Takeda N, Sato G, et al. Changes in slow phase eye velocity and time constant of positional nystagmus at transform from cupulolithiasis to canalolithiasis. Acta Otolaryngol 2008;128:22-8.

6. Bergenius J, Tomanovic T. Persistent geotropic nystagmus-a different kind of cupular pathology and its localizing signs. Acta Otolaryngol 2006;126:698-704

7. Imai T, Takeda N, Morita M, et al. Rotation vector analysis of eye movement in three dimensions with an infrared CCD camera. Acta Otolaryngol 1999;119:24-8.

8. Haslwanter T. Mathematics of three-dimensional eye rotations. Vision Res 1995;35:1727-39.

9. Imai $\mathrm{T}$, Sekine $\mathrm{K}$, Hattori $\mathrm{K}$, et al. Comparing the accuracy of video-oculography and the scleral search coil system in human eye movement analysis. Auris Nasus Larynx 2005;32:3-9.

10. Raphan T. Modeling control of eye orientation in three dimensions. I. Role of muscle pulleys in determining saccadic trajectory. J Neurophysiol 1998;79:2653-67.

11. Schnabolk C, Raphan T. Modeling three-dimensional velocity-to-position transformation in oculomotor control. J Neurophysiol 1994:71:623-38.

12. Arzi M, Magnin M. A fuzzy set theoretical approach to automatic analysis of nystagmic eye movements. IEEE Trans Biomed Eng 1989;36:954-63.

13. Naoi K, Nakamae K, Fujioka $\mathrm{H}$, et al. Three-dimensional eye movement simulator extracting instantaneous eye movement rotation axes, the plane formed by rotation axes, and innervations for eye muscles. IEICE Trans Inf Syst 2003;11:2452-62.

14. Imai T, Masumura C, Takeda N, et al. Pseudo-anterior canalolithiasis. Acta Otolaryngol 2013;133:594-9.

15. Ichijo $\mathrm{H}$. Persistent direction-changing geotropic positional nystagmus. Eur Arch Otorhinolaryngol 2012;269:747-51.

16. $\mathrm{Kim} \mathrm{CH}, \mathrm{Kim} \mathrm{MB}, \mathrm{Ban} \mathrm{JH}$. Persistent geotropic direction-changing positional nystagmus with a null plane: the light cupula. Laryngoscope 2014;124:E15-19. 